\title{
High Identification Rates of Endogenous Neuropeptides from Mouse Brain
}

\author{
Xiaozhe Zhang, ${ }^{* \dagger}$ Filomena Petruzziello, $^{\dagger}$ Fabio Zani, ${ }^{\dagger}$ Laetitia Fouillen $^{\dagger}$ Per E. Andren, ${ }^{\ddagger}$ \\ Giovanni Solinas, ${ }^{\dagger}$ and Gregor Rainer ${ }^{\dagger}$ \\ ${ }^{\dagger}$ Department of Medicine, University of Fribourg, Fribourg, CH-1700, Switzerland \\ ${ }^{*}$ Department of Pharmaceutical Biosciences, Medical Mass Spectrometry, Uppsala University, Biomedical Centre, Box 591, SE-75124 \\ Uppsala, Sweden
}

\begin{abstract}
Mass spectrometry-based neuropeptidomics is one of the most powerful approaches for identification of endogenous neuropeptides in the brain. Until now, however, the identification rate of neuropeptides in neuropeptidomics is relatively low and this severely restricts insights into their biological function. In the present study, we developed a high accuracy mass spectrometry-based approach to enhance the identification rates of neuropeptides from brain tissue. Our integrated approach used mixing on column for loading aqueous and organic extracts to reduce the loss of peptides during sample treatment and used charge state-directed tandem mass spectrometry to increase the number of peptides subjected to high mass accuracy fragmentation. This approach allowed 206 peptides on average to be identified from a single mouse brain sample that was prepared using $15 \mu \mathrm{L}$ of solutions per $1 \mathrm{mg}$ of

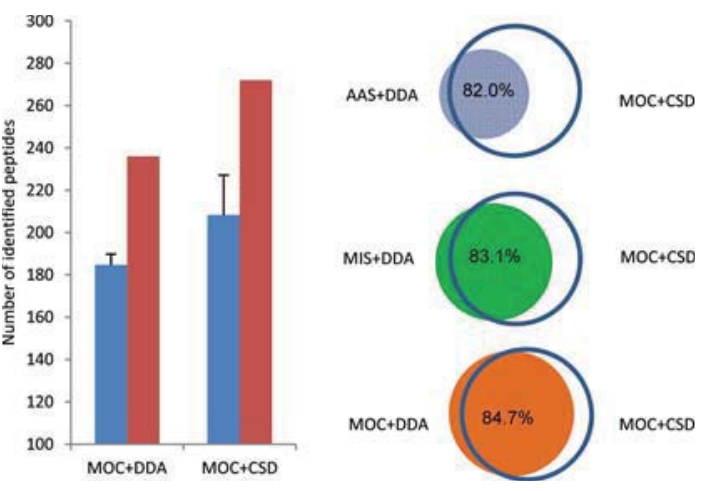
tissue. In total, we identified more than 500 endogenous peptides from mouse hypothalamus and whole brain samples. Our identification rate is about two to four times higher compared to previously reported studies conducted on mice or other species. The hydrophobic peptides, such as neuropeptide $\mathrm{Y}$ and galanin, could be presented and detected with hydrophilic peptides in the same LC-MS run, allowing a high coverage of peptide characterization over an organism. This will advance our understanding of the roles of diverse peptides and their links in the brain functions.
\end{abstract}

KEYWORDS: neuropeptidomics, neuropeptide, identification rate, charge state-directed tandem MS

\section{INTRODUCTION}

Neuropeptides, including endogenous peptide neuromodulators and hormones, are involved in diverse physiological and behavioral processes, such as food intake, sleep and reward processing. ${ }^{1}$ Neuropeptides are produced from neuropeptide precursors under the selective action of endopeptidases, and some of them undergo further posttranslational modifications (PTMs), which can profoundly influence their bioactivity. Individual peptides in general have distinct biological functions, even though they are derived from the same prohormone precursor. It is thus important to identify with high confidence as many peptides as possible derived from particular prohormone precursors, to understand the biological functions.

Mass spectrometry (MS)-based neuropeptidomics, a subbranch of proteomics, has now emerged as the method of choice for the sequencing of endogenous neuropeptides in biological samples. ${ }^{2-4}$ An explicit goal of neuropeptidomics is the complete characterization of the neuropeptides, including classical neuropeptides and potentially bioactive peptide fragments derived from prohormone precursors, in a targeted organism. Neuropeptides are endogenous peptides of various lengths and thus different hydrophobicities. This diversity leads to a generally low identification rate in neuropeptidomics. Until now, the identification rate is believed to only cover a small fraction of the peptides derived from neuropeptide precursors. ${ }^{4,5}$ This is evident because in any single study, many classical neuropeptides, which are known to be present in the brain, remain undetected. This low overall identification rate restricts possibilities for the discovery of neuropeptide functions in biological studies. ${ }^{5}$

A number of studies have addressed the discovery of as many neuropeptides as possible from brain samples by using various methods such as novel sample treatment methods, 6,7 derivatization, ${ }^{4}$ two dimension separation, ${ }^{8}$ high resolution MS, ${ }^{9,10}$ and database construction. ${ }^{11}$ This work has substantially improved the neuropeptide identification rate and greatly enlarged our knowledge of neuropeptides in brain. Previous single studies have generally been able to report around 50150 peptides, including but a few classical neuropeptides, regardless of whether the sample was prepared from a specific brain area or the whole brain. Current studies showed that in 
the brain, signaling molecules work in a concerted manner to maintain the brain functions. ${ }^{12}$ Therefore, a high identification rate of neuropeptides is of great interest in the field of basic neuroscience and pharmaceutical research, because it could provide a more comprehensive understanding of the functional roles of various neuropeptides in the brain.

Neuropeptidomics typically require several serial procedures, including tissue preparation, peptide extraction, tandem MS (MS/MS) data acquisition and data interpretation, to perform a comprehensive identification of peptides. The peptide identification rate thus depends critically on the success of each procedure. Leaving restrictions due to bioinformatics aside ${ }^{13}$ we found that the low identification rate of neuropeptides could be caused mainly by two issues: (i) the pronounced loss of peptides during sample treatment and (ii) the loss of the tandem MS information of many peptides, in particular low abundance ones, during data acquisition. We reasoned that the reduction of such losses would enable the significant enhancement of the peptide identification rate.

In this study, we have developed a new approach to increase the identification rate of endogenous peptides from brain extracts. Our integrated approach used mixing on column (MOC) for sample treatment and used charge state-directed (CSD) tandem MS to increase the number of peptides subjected for high mass accuracy fragmentation. Our approach used a separation-and-pool mode at both the sample treatment and data processing stages. It contains several key methods, which include mixing on column (MOC) sample treatment, charge state-directed (CSD) liquid chromatography (LC)Fourier transform (FT)-MS/MS, and hybrid spectral analysis. Using our integrated approach, more than 500 endogenous peptides in total were identified from mouse hypothalamus and whole brain samples, demonstrating previously unreached identification power in neuropeptidomics.

\section{EXPERIMENTAL PROCEDURES}

\section{Materials}

LC-MS grade acetonitrile and formic acid were purchased from Fisher Scientific (New Jersey, USA) and Fluka (Wisconsin, USA), respectively. Acetic acid was purchased from Fluka (Buchs, Switzerland). Pure water was prepared by GenPure system (TKA, Niederelbert, Germany). Siliconized microcentrifuge tubes $(2 \mathrm{~mL})$ were purchased from Eppendorf (Hamburg, Germany). Microcon centrifugal filter devices (Vivacon 500) were purchased from Sartorius AG (Goettingen, Germany).

\section{Animals}

An inbred strain of black mice (C57BL/6, $n=8$ ) were used in the experiments. Animals were housed in a standard facility with a $12 \mathrm{~h}$ light/dark cycle under constant temperature and humidity. Mice were fed with normal chow diet, and they were given free access to food and water. All procedures with live animals were conducted with protocols approved by the veterinary office of Fribourg, Switzerland.

\section{Brain Tissue Preparation}

The degradation of proteins or neuropeptides themselves, which occurs during the post-mortem period, ${ }^{14,15}$ greatly influences the qualitative results and also affects the reproducibility in quantitative analysis. ${ }^{14,16,17}$ In all the sample preparation experiments, each step was under temperature control to minimize the degeneration of the endogenous peptides and also to reduce the amount of interference peptides produced from fragmentation of proteins. ${ }^{2,14}$ Male mice of 810 weeks old were sacrificed, and then their brains were removed immediately and heat stabilized using Denator irradiation (Denator AB, Gothenburg, Sweden) as described elsewhere. ${ }^{7}$ Three mouse brains were pooled and homogenized as entire brain (EB) samples for method comparison. Three samples prepared using the MOC method were further subjected to LC-FT-MS/MS analysis for identification of peptides from EB. Another five mouse brains were used only for identification of peptides from the hypothalamus. The hypothalami of both hemispheres from each brain were dissected at $-10^{\circ} \mathrm{C}$, pooled as one sample, and then submitted for extraction.

\section{Extraction of Peptides}

To acquire the maximum extraction efficiency for diverse peptides of different hydrophobicities, three different extraction methods, acetic acid solution (AAS), mixing in solution (MIS), and mixing on column (MOC), were examined. AAS sample treatment method was as follows: each piece of homogenized EB was extracted two times with $0.2 \%$ acetic acid aqueous solution. Each step used $7.5 \mu \mathrm{L}$ of solution per $1 \mathrm{mg}$ of tissue. The two-step extractions were mixed and submitted to filtration. This method has been shown to have high reproducibility in peptide quantitative analysis. ${ }^{18,19}$ MIS sample treatment method was as follows: each piece of homogenized EB was extracted three times using (i) water-methanol-acetic acid solution (89.8:10:0.2, v/v/v), (ii) water-methanol-acetic acid solution (69.8:30:0.2, v/v/v), and (iii) water-methanolacetic acid solution (49.8:50:0.2, v/v/v), respectively. ${ }^{10}$ Each step used $5 \mu \mathrm{L}$ of solution per $1 \mathrm{mg}$ of tissue. Before filtration, all the three extractions were mixed directly. MOC sample treatment method was as follows: each piece of homogenized EB was extracted four times using (i) $0.2 \%$ acetic acid aqueous solution, (ii) $0.2 \%$ acetic acid aqueous solution, (iii) watermethanol-acetic acid solution (79.8:20:0.2, v/v/v), and (iv) water-methanol-acetic acid solution (49.8:50:0.2, v/v/v), respectively. Each step used $3.75 \mu \mathrm{L}$ of solution per $1 \mathrm{mg}$ of tissue. Before filtration, the first two step aqueous extractions and the last two step organic extractions were mixed, respectively. The last two steps used methanol to increase the capability in the extraction of hydrophobic peptides.

In each extraction step of all the three extraction methods, every piece of tissue was homogenized twice (each time $20 \mathrm{~s}$ with a pause of $20 \mathrm{~s}$ between the homogenizations) for $1 \mathrm{~min}$ by a Precellys 24 homogenizer (Bertin Technologies, Montigny-le-Bretonneux, France). Then, the sample was centrifuged at $22000 \mathrm{~g}$ for $60 \mathrm{~min}$ at $4{ }^{\circ} \mathrm{C}$. All the supernatants obtained in the extraction methods were filtered on a $10 \mathrm{kDa}$ cutoff filter (Vivacon 500, Sartorius AG, Goettingen, Germany) by centrifuging for $90 \mathrm{~min}$ at $14000 \mathrm{~g}$ at $4{ }^{\circ} \mathrm{C}$.

\section{Comparative Analysis of Three Sample Preparation Methods}

Three pooled, homogenized entire brains (EB) were used for comparative analysis between the three sample treatment methods. The test of each method used three EB samples (30 $\mathrm{mg} /$ sample) with two replications of LC-FT-MS analysis. Each analysis was conducted on a nanoLC-MS system (see details in LC-FT-MS/MS Data Acquisition). The LC parameters were identical to those used for LC-FT-MS/MS data acquisition except for the use of a short elution gradient. The gradient profile was as follows: $0-3.5 \mathrm{~min}, 2 \% \mathrm{~B}$; $3.5-8 \mathrm{~min}, 2-25 \% \mathrm{~B}$; 
$8-32 \mathrm{~min}, 20-50 \% \mathrm{~B} ; 32-38 \mathrm{~min}, 50-70 \% \mathrm{~B} ; 38-40 \mathrm{~min}$, 95\% B. After each LC-MS sample analysis, the LC system was cleaned by injection of $75 \%$ acetonitrile $(0.2 \% \mathrm{FA})$ one or two times and conditioned for 10 min with $50 \% \mathrm{~B}$. The MS signals were acquired using FT-MS without fragmentation events.

The software SIEVE (Thermo Scientific, USA) was used to extract the peak areas of each peptide across different samples. It served as a data alignment and analysis tool with following parameters: $\mathrm{m} / z$ range from 300 to $1500 \mathrm{Da}$, time frame $5 \mathrm{~min}$, $\mathrm{m} / z$ frame $0.01 \mathrm{Da}$ with a peak intensity threshold 100000 . [D$\mathrm{Tyr}^{27,36} \mathrm{D}-\mathrm{Thr}^{32}$ ]-Neuropeptide Y (AA27-36) was used as internal standard for quality control. The normalized intensities of the 2000 most intensive features were plotted against their corresponding retention time (Rt). The average intensities (integrated peak areas) of each feature in AAS, MIS, and MOC samples were summarized and accounted as a reference (100\%). The average intensity of this feature in AAS, MIS, or MOC samples was then normalized to the reference value.

\section{LC-FT-MS/MS Data Acquisition}

The peptide extracts were analyzed using a LTQ-Orbitrap Discovery (Thermo Fisher Scientific, Bremen, Germany) coupled with a 2D NanoLC (Eksigent Technologies, California, USA). In each LC-MS run, $5 \mu \mathrm{L}$ of sample was injected with 1D pump to a trap column (100 $\mu \mathrm{m}$ ID, $2 \mathrm{~cm}$ long), which was packed with a C18 AQ particles $(5 \mu \mathrm{m}, 100 \AA$, Dr. Maisch $\mathrm{GmbH}$, Ammerbuch-Entringen, Germany) in a PEEK column holder (Upchurch, Oak Harbor, WA, USA). For each sample prepared in MOC, the organic extract was loaded on the trap column $3 \mathrm{~min}$ prior to the injection of the aqueous extract. Prior to injections, the trap column was conditioned using $2 \%$ acetonitrile and $98 \%$ water containing $0.2 \%$ formic acid. The elution direction of trap column was reversed through a 10-port valve when it started to couple with the analytical column. The analytical column of $25 \mathrm{~cm}$ long used C18 AQ particles $(3 \mu \mathrm{m}$, $100 \AA$ ) as medium, which was packed in a Picofrit capillary with an emitter tip of $10 \mu \mathrm{m}$ (New Objective, Woburn, MA, USA). The mobile phase A and B in 2D pump (Chanel 2) were $0.2 \%$ FA, and $95 \%$ acetonitrile ( $0.2 \% \mathrm{FA})$, respectively.

The gradient profile was as follows: $0-6 \mathrm{~min}, 2 \% \mathrm{~B} ; 6-12$ $\min , 2-20 \% \mathrm{~B} ; 12-80 \mathrm{~min}, 20-50 \% \mathrm{~B} ; 80-85 \mathrm{~min}, 50-60 \%$ B; $85-90 \mathrm{~min}, 60-95 \% \mathrm{~B}$; and $90-100 \mathrm{~min}, 95 \% \mathrm{~B}$. After each LC-MS sample analysis, the LC system was cleaned by injection of $75 \%$ acetonitrile ( $0.2 \% \mathrm{FA})$ one or two times and conditioned for $10 \mathrm{~min}$ with $50 \% \mathrm{~B}$.

Data dependent acquisition (DDA)-LC-FT-MS/MS: Data acquisition on the LTQ-FTMS instrument consisted of a full FTMS scan event at mass range of $350-2000 \mathrm{~m} / z$ and datadependent collision-induced dissociation (CID) MS/MS scans (30000 resolution) of the five most abundant peaks from the previous full FTMS scan. The mass resolution for each scan event was kept at 30000 . Mass spectrometric experimental parameters were selected to provide high quality fragmentation spectra of the majority from the possible compounds of interest. Minimum signal threshold for the first and fifth most intensive ions were selected to 100000 counts, respectively; isolation width was selected to $m / z=2$; maximum accumulation time is $300 \mathrm{~ms}$, normalized collision energy, 30\%; activation Q 0.25; and activation time, $50 \mathrm{~ms}$, Dynamic exclusion was set as a repeat count of 1 , an exclusion duration of $30 \mathrm{~s}$, and a repeat duration of $30 \mathrm{~s}$. Dynamic exclusion used $25 \mathrm{ppm}$ mass tolerance.
CSD-LC-FT-MS/MS: The selection of charge state 1, 2, 3, $\geq 4$ was conducted by using the charge rejection function in the Xcalibur software. To select the charge state of interest, the options for the rejection of other charge states are enabled. In this manner, four CSD-LC-FT-MS/MS analyses were conducted for each sample by the instrument automatically selecting the peptides of directed charge state(s) for fragmentation. The Xcalibur software does not allow specifically selection of peptides with charge $\geq 4$. To compensate, the inclusion list of peptides of charge 4 or 5 in the previous CSDLC-FT-MS/MS analysis was prepared, respectively. Software Sieve (1.3 version, Thermo, CA, USA) was used to extract the features with charge states 4 or 5 from raw LC-FT-MS/MS data. The inclusion mass lists were then loaded to the FT-MS/ MS scan for another two CSD-LC-FT-MS/MS runs for each sample.

\section{Hybrid Spectral Analysis}

All the raw LC-FTMS/MS data were subjected to Peaks Studio 5.3 (BSI, Canada) for spectral interpretation. ${ }^{20,21}$ Database search was conducted against the Swepep mouse neuropeptide precursors database. ${ }^{11}$ The Swepep precursor database was downloaded from www.swepep.org and loaded to Peaks studio 5.3 by using the UniprotKB/TrEMBL FASTA format and taxonomy options. Peaks Studio 5.3 was used for various functions such as Data Refinement, Auto De Novo, and Peaks Search (homology search). ${ }^{22}$ The Data Refinement program allows correcting of the precursor mass and charge states to provide the accurate monoisotopic mass of a peptide. Data processing, including peak centroiding, charge deconvolution, and deisotope, was conducted for data refinement. The refined data were subjected to database search with the mass tolerance of precursor ions and product ions set at $10 \mathrm{ppm}$ and $0.05 \mathrm{Da}$, respectively. No enzyme was specified for cleavage. Variable PTMs, including amidation (C-terminal), acetylation (Nterminal), phosphorylation, pyroglutamalytion from glutamatic acid and glutamine ( $\mathrm{N}$-terminal), were selected in sequencing.

Estimation of false positives was conducted by searching all spectra against decoy databases. The cutoff of PDR (peptide sequence) for peptide identification in Peaks search was $<1 \%$. The peptides of FDR between $\geq 1 \%$ but $-10 \log P>20$ were submitted to manual inspection to confirm their sequences. A sequence was considered correct only if it matched all the following criteria: (1) the mass of a peptide must have been calculated from the monoisotopic ions of a peptide, (2) all of the database search results were inspected with de novo sequencing results, (3) the peptide mass was within $10 \mathrm{ppm}$ of the theoretical mass, (4) the major fragments observed in MS/ MS had to match within $0.05 \mathrm{Da}$ to predicted monoisotopic production ions, and (5) the fragmentation information must be enough to recognize the alignment of amino acids, in particular if they fall in the substitution positions across the adjacent species.

\section{RESULTS}

\section{Enhanced Peptide Extract and Load Efficacy with Mixing-on-Column Sample Treatment}

We first examined the influence of different sample treatment methods on peptide extraction efficacy. The intensities (peak areas) of each feature (peptide) were compared across the samples prepared with three methods, AAS, MIS, and MOC. The normalized intensities of the 2000 most intensive features were plotted against their corresponding retention time (Rt) 

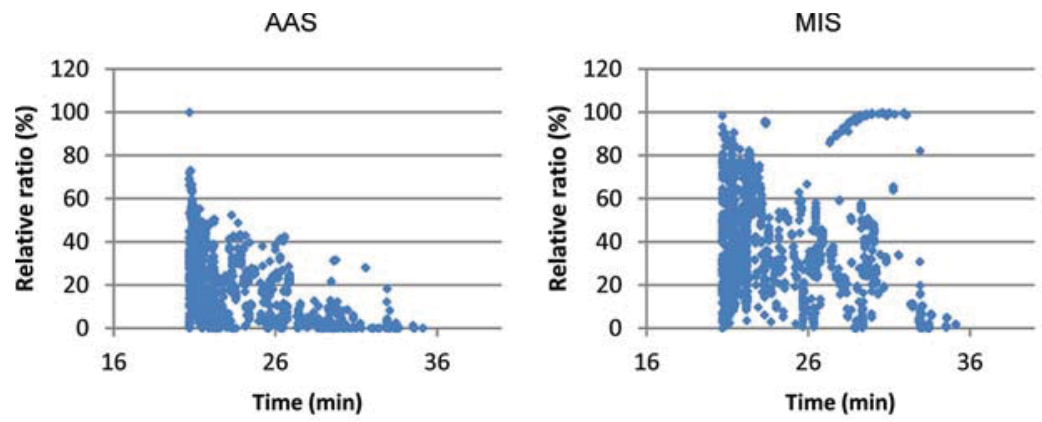

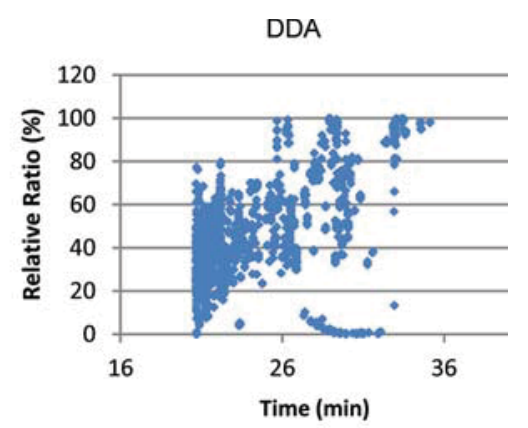

(A)

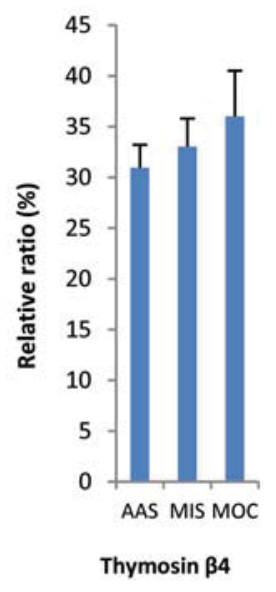

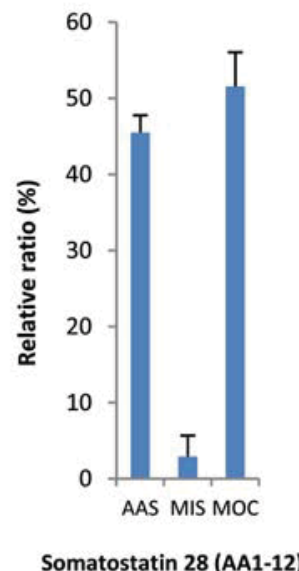

Somatostatin 28 (AA1-12)
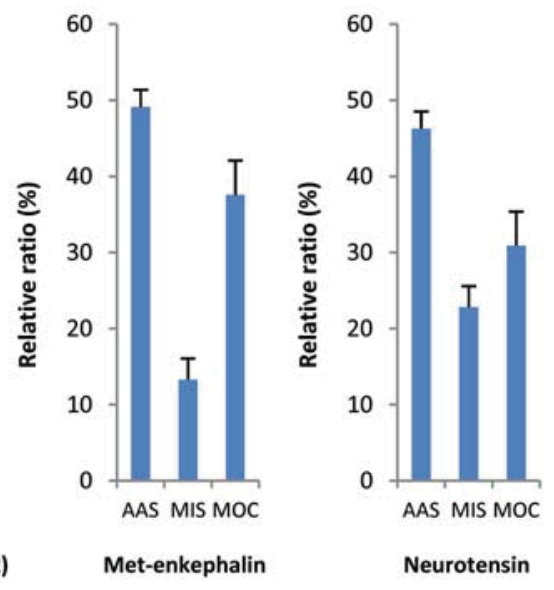

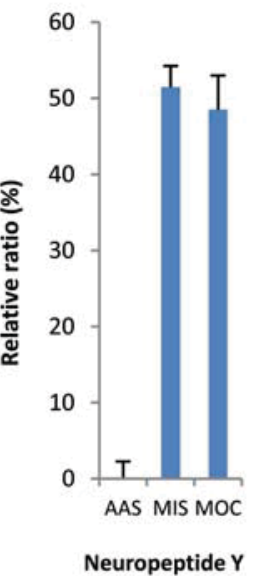

(B)

Figure 1. The relative intensity of features between the AAS, MIS, and MOC methods. (A) The normalized intensities of 2000 features are plotted against their retention time for brain samples prepared using AAS, MIS, and MOC. (B) The intensities of four representative peptides of different retention times, plus a high abundance reference peptide thymosin $\beta$. The bars indicate the mean relative intensities \pm s.e.m $(n=6)$.

(Figure 1A). The three methods show remarkable difference in their intensity-Rt patterns. The AAS method had overall low extraction efficacy, while the MOC method in general allowed high extraction efficacy. In AAS samples, the number of features with relative intensity $>33.3 \%$ decreased with the increase of retention time, and plenty of features were almost undetectable (relative intensity close to $0 \%$ ). In MOC samples, the number of features with relative intensity $>33.3 \%$ (1330) is larger than those in AAS samples (279) and MIS (1035) samples. Compared to MIS samples, MOC samples had much fewer features with relative intensities close to $0 \%$. In contrast, their features had moderate to high relative intensities across the whole time course. The overall intensity-Rt pattern indicated that the MOC method allowed a large number of peptides to be extracted and loaded to LC-MS analysis with high coverage and high abundance.

The examination on individual peptides further verified the high extraction efficacy of the MOC methods (Figure 1B). Thymosin $\beta 4$, a polar peptide hormone of high abundance, was used as a reference peptide in comparative analysis. The relative intensities of this peptide were similar across the three methods. In a sharp contrast, somatostatin-28 (AA1-12), met-enkephalin, neurotensin, and neuropeptide $\mathrm{Y}$ were unequally extracted and loaded for LC-MS analysis. Somatostatin-28 (AA1-12), a polar peptide, had much more lower abundance in MIS samples than in AAS and MOC samples, while neuropeptide $\mathrm{Y}$ was well extracted in MIS and MOC samples but not in AAS samples. Compared to the MOC method, the direct mixing of aqueous and organic extracts before filtration and loading also led to the abundance reduction of peptides somatostatin 28 (AA1-12) and neuropeptide $Y$. These observations indicated that the extraction efficacy of a peptide heavily depended on the used solutions and procedure. As the quality of the tandem MS spectra of a peptide depends on its abundance, the use of the MOC method could increase the peptide identification rate because of its high coverage of peptides of different hydrophobicities.

\section{Charge State-Directed High Accuracy Tandem MS Data Acquisition}

In the experiments, we carried out a set of CSD-LC-FT-MS/ MS analyses $(n=6)$ for each sample to acquire high accuracy tandem MS information (see the Experimental Procedures). After the six LC-MS/MS analyses, all the data from a given sample are pooled by spectral analysis software and submitted for further hybrid spectral analysis. The database search was conducted on the sample level (for the six LC-FT-MS/MS data) rather than an individual LC-FT-MS/MS data. The software then gave a list of peptides identified from these raw data. The manual work required for data processing is therefore minimized when using a set of CSD-LC-MS/MS analysis for a sample. 
To evaluate the performance of our integrated approach, we compared the number of peptides identified from mouse entire brain (EB) using different LC-FT-MS/MS methods and different sample treatment methods. Overall, our new approach allowed 206 peptides on average to be identified from a single mouse brain sample, which was prepared using $15 \mu \mathrm{L}$ of solutions per $1 \mathrm{mg}$ of tissue. The cumulative number of peptides identified from three EB samples reached a value of 272. Figure 2A shows that the CSD LC-FT-MS/MS method

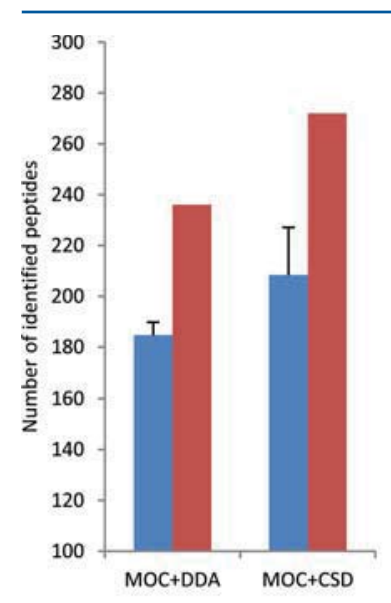

(A)

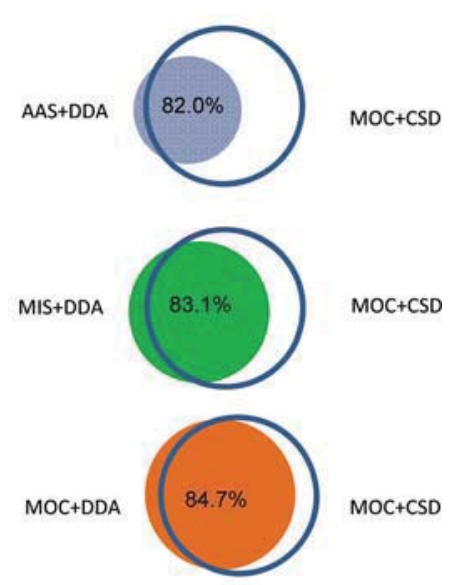

(B)
Figure 2. Comparison of different neuropeptidomic approaches. (A) The number of peptides identified with different methods. (B) The overlap of identified peptides between different methods. The homogenized entire brain (EB) samples are analyzed using different LC-FT-MS/MS analysis methods (DDA or CSD) with different sample treatment methods (AAS, MIS, and MOC). Each sample is analyzed using six LC-MS/MS runs, and each method is examined with three brain tissue samples. The bars indicate the average numbers (mean \pm s.e.m) of identified peptides per EB sample (blue) and the sum of unique identified peptides from three EB samples (red). All the EB samples used for method comparison are prepared from the homogenized tissues of three pooled mouse brains. The peptide sequence FDR is less than $1 \%$.

allows more peptides, no matter counting in average and cumulative numbers, to be identified than the DDA LC-FTMS/MS method. Further comparative analysis shows that our new approach allowed more peptides to be identified than DDA LC-FT-MS/MS analysis combined with the commonly acetic acid solution (AAS) sample treatment method ${ }^{2,23}$ or mixing in solution (MIS) multistage extraction method. ${ }^{10}$ The results showed that about $82.0 \%$ of the peptides identified using method AAS +DDA had sequences identical with those using MOC+CSD. The peptides identified using method MIS+DDA and MOC+DDA had 83.1 and $84.7 \%$ sequences identical with those using $\mathrm{MOC}+\mathrm{CSD}$, respectively (Figure $2 \mathrm{~B}$ ). The results demonstrated the peptide identification rate is method dependent, while our new approach allowed an overall high overlap of identified peptides with other approaches.

Prohormone-Derived Peptides Identified from Moue Brain

Overall High Peptide Identification Rate from Mouse Whole Brain and Hypothalamus Samples. When we applied our integrated approach on samples taken from the mouse hypothalamus, a peptide-rich brain region, we were able to identify 367 peptides from neuropeptide precursors. In total, 457 unique peptides were identified from both the homogen- ized EB and hypothalamus of wild type mice at confidence level peptide sequence FDR (false discovery rate) $<1 \%$. An additional 46 peptides were identified by manual inspection on other peptides (FDR $\geq 1 \%$ but $-10 \log P>20)$ using strict criteria (see the Experimental Procedures). ${ }^{9}$ Overall, 503 unique peptides were identified from mouse brain (see Supporting Information Table S1).

Identification of Long and Hydrophobic Peptides. The brain expresses various neuropeptides with long sequence and high hydrophobicity. We unambiguously identified a number of long neuropeptides, such as galanin, orexin-B, neuropeptide $\mathrm{Y}$, CLIP, and oxyntomodulin. The use of FT-MS/MS showed that peptides can be identified in high confidence. Figure 3 represents the identification of glucagon-like peptide $1(7-36)$ and neuropeptide Y. Additionally, our new approach allows a number of high hydrophobic peptides to be identified along with polar peptides. Figure 4 shows representative high abundance peptides identified from one mouse brain (EB) sample. The base peak LC-MS chromatography indicated that peptides of different hydrophobicity, including polar somatostatin $28(\mathrm{AA} 1-12)$ and hydrophobic secretoneurin, could be extracted, loaded, and detected in a single LC-MS run.

Discovery of Novel Peptides. The high identification rate of our integrated approach allowed the discovery of a large number of novel peptides $(n=189)$ that have not been previously reported in mouse or other species (see Supporting Information Table S1). Figure 5 represents an example of spectral identification for a novel peptide, $Q(-17.03)$ LRAPGAMLQIEALQEVLKKLKS, derived from precursor cocaine-and-amphetamine-regulated transcript (CART). This peptide has classical dibasic cleavage sites and is thus a possible candidate for a bioactive neuropeptide. Further experimental work is required to gain insight into the biological functions of this and other newly discovered peptides.

Identification of Peptides with PTMS. Post translational modifications (PTMs) commonly occur in neuropeptides and have important biological functions. They can alter functional properties of neuropeptides, for example, increasing the binding affinity to receptors or make peptides more resistant to enzymatic degradation. ${ }^{1}$ In the present study, we characterized 69 peptides containing PTMs (see Supporting Information Table S1), which include 6 phosphorylations, 7 acetylations, 18 pyruglutamations, and 41 amidations. Some of the peptides exhibited more than one PTMs. For example, both pyroglutamylation and amidation modifications were detected on vasoactive intestinal peptide (VIP), Q(-17.03)MAVKKYLNSILN(-0.98). The PTM of C-terminal amidation, which is specific to endogenous peptides ${ }^{24}$ and is required for the functional activation of many neuropeptides, ${ }^{25}$ occurred most frequently in our data set.

\section{DISCUSSION}

The improvement in the identification rate of endogenous peptides is a major current goal of neuropeptidomics. Our study developed an integrated approach to increase the peptide identification rate. This approach used mixing on column (MOC) to increase the peptide loaded onto the column and used charge state-directed (CSD) tandem MS to increase the number of peptides subjected for high mass accuracy fragmentation. Using our integrated approach, more than 500 endogenous peptides in total were identified from mouse hypothalamus and whole brain samples, demonstrating previously unrivaled identification power in neuropeptidomics. 


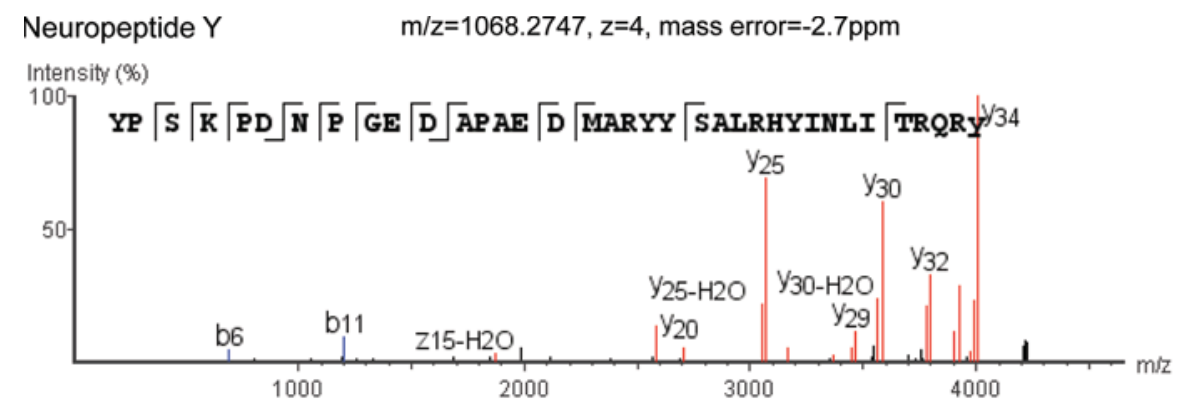

(A)

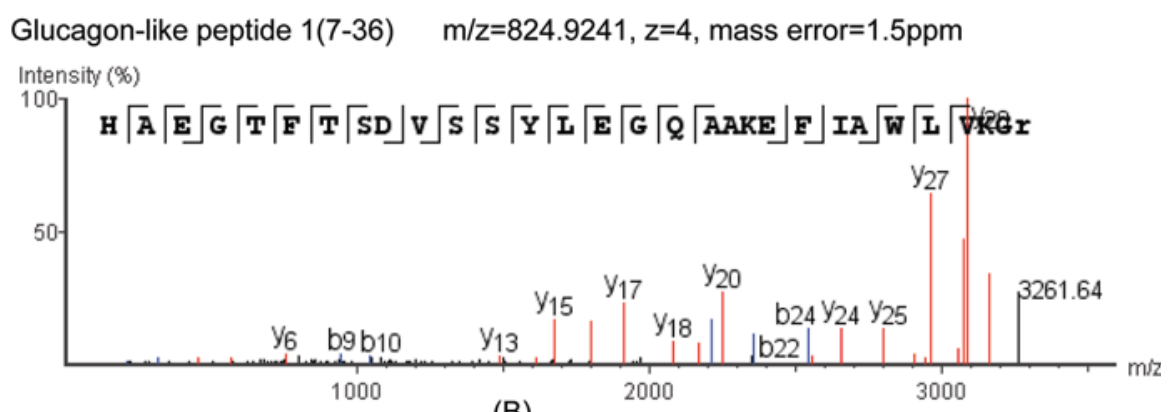

(B)

Figure 3. Annotated FT-MS/MS spectra of representative peptides neuropeptide Y (A) and glucagon-like peptide 1(7-36) (B). The high accuracy of the MS and MS/MS information allows these peptides to be identified with high confidence.

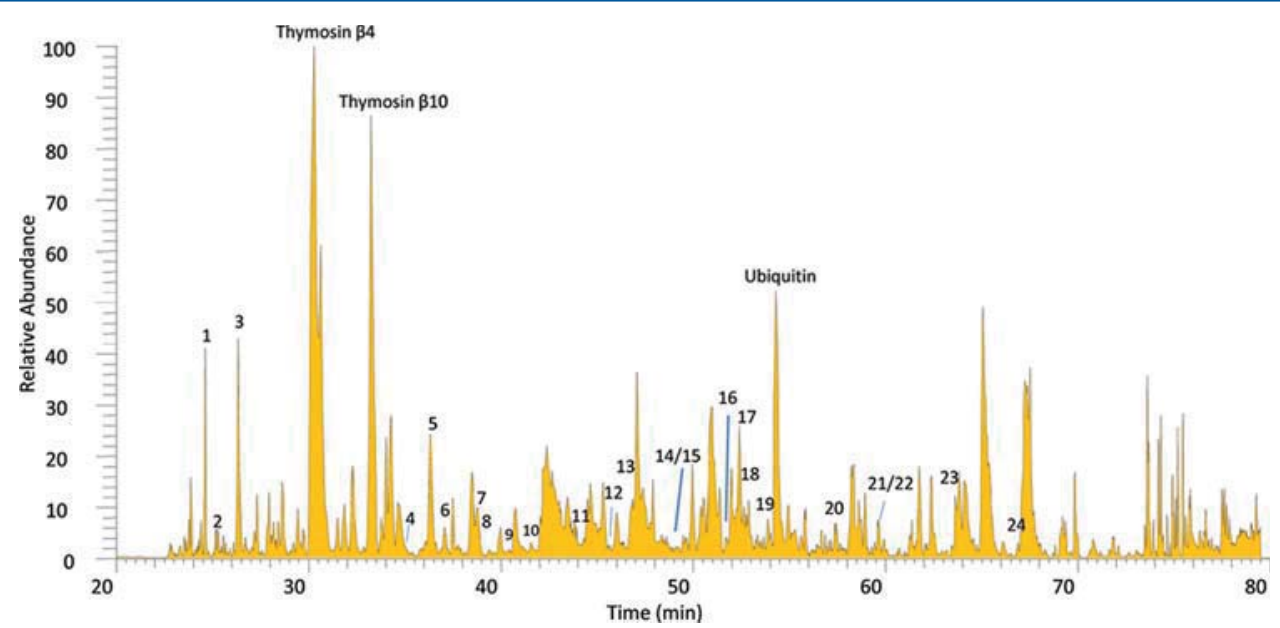

Figure 4. Representative prohormone-derived peptides identified from a mouse brain (EB) sample. These peptides include somatostatin-28-14 (1), nociceptin (2), cerebellin-1 (3), dynorphin B (4), neurokinin A (5), neuropeptide-glutamic acid-isoleucine (6), met-enkaphalin (7), neurotensin (8), somatostatin-28 (9), met-enkephalin-arg-ser-leu (10), galanin (11), leu-enkaphalin (12), vasoactive intestinal peptide (13), WE-14 (14), BAM18 (15), oxyntomodulin (16), little SAAS (17), somatostatin-14 (18), manserin (19), neuropeptide Y (20), neurokinin-B (21), GAV (22), PEN (23), and secretoneurin (24). These peptides are detected with high MS signals (intensity larger than 1E6). One small protein, ubiquitin, and two peptide hormones, thymosin $\beta 4$ and thymosin $\beta 10$, are used in practice as reference peptides to monitor the elution order of peptides.

Sample quality critically influences the data output in peptidomics and proteomics. $^{26}$ Neuropeptides are a large family of compounds with a wide range of hydrophobicities, including strongly hydrophobic and hydrophilic molecules. This diversity of hydrophobicities makes it difficult to extract and preserve diverse neuropeptides in a single solution, such as the commonly used aqueous buffers. ${ }^{2,27}$ The extraction procedure therefore has a crucial influence on the number and amount of peptides delivered to the LC-MS/MS analysis. ${ }^{6}$ Remarkable losses of peptides in sample treatment have been previously observed during preservation. ${ }^{28}$ Our study also showed that some peptides can be lost during direct mixing of different solutions in a multistage extraction (see Supporting
Information Figure S1). We therefore used a MOC method to sequentially extract and load peptides with aqueous and organic solutions to increase the coverage of both hydrophilic and hydrophobic peptides. As the hydrophilic peptides have low retention times and thus can be easily washed out from trap column with high content of organic solvents used for samples, the load of an organic extract was conducted prior to the load of an aqueous extract. Using these procedures, both hydrophilic and hydrophobic peptides were well extracted and loaded for further LC-MS analysis.

Our study increased the peptide identification rate with fully using the advantage of high accuracy tandem MS. Many of the endogenous peptides have long sequences, for example, 
CART(AA57-79): RR.Q(-17.03)LRAPGAMLQIEALQEVLKKLKS.KR

$\mathrm{m} / \mathrm{z}=637.6224, \mathrm{z}=4$, mass error $=0.4 \mathrm{ppm}$

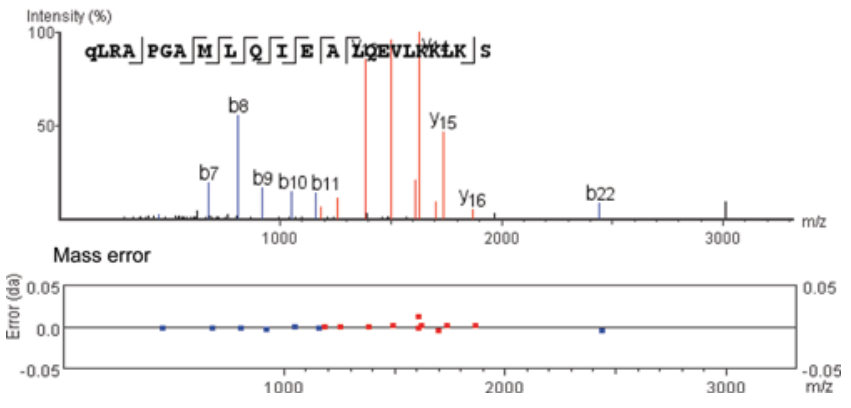

Figure 5. Identification of a novel hydrophobic peptide CART (AA57-79). This peptide, RR.Q(-17.03)LRAPGAMLQIEALQEVLKKLKS.KR, has dibasic cleavage sites and thus can be a candidate neuropeptide.

neuropeptide $\mathrm{Y}$ with 36 amino acid residues, which increases the difficulty of sequence identification. Recent studies have shown that the application of high resolution (HR) instruments, such as LTQ-Orbitrap, significantly narrow the search space in databases, and serve as a powerful approach in peptide discovery. ${ }^{9,10,29,30}$ To obtain tandem MS information for as many peptides as possible, we acquired high accuracy tandem MS by using CSD LC-FT-MS/MS, which was adapted from a newly developed directed LC-MS/MS method ${ }^{10,31}$ but used charge states to guide the tandem MS analysis. Compared to peptides produced from trypsin digestion, neuropeptides are endogenous peptides of various lengths, of which the charge states in electrospray ionization (ESI)-MS range from 1 to 5 or even higher. Commonly observed in a LC-MS analysis, several peptides with different charge states coelute at the same time window. Our approach method allows peptides, including low abundance ones, to be selected by software (Xcalibur in our case) for fragmentation according to the charge states of their precursor ions. The use of charge state directed LC-FT-MS/ MS analysis thus compensates for the low scan speed of LTQOrbitrap MS, ${ }^{32}$ allowing the acquisition of a larger number of high quality fragmentation spectra for the peptides present in samples.

It is worth noting that in the present study, we stabilized mouse brain tissue by using a novel method, which utilizes a combination of heat and pressure under vacuum to stop the degradation of brain tissue immediately after extracting brains from animals. A line of evidence has demonstrated that proteases and other protein-modifying enzymes can rapidly change the composition of the proteome following brain extraction, leading to extensive changes of the proteome. ${ }^{7,14,33-36}$ Post-mortem changes of the brain cause major disturbance in the tissue homeostasis, which in turn causes rapid release of mediators of degradation, altering the levels and composition of proteins and post-translational modifications (PTMs). ${ }^{5,14}$ The application of rapid tissue stabilization has been shown to substantially reduce protein fragmentation interference, and importantly, to maintain the levels of neuropeptides in the brain tissue. $5,7,14,37$

Our study allowed identifying a large number of unreported peptides, which included peptides with $\mathrm{N}$ - and C-terminal modifications and/or dibasic cleavage sites as well as peptide fragments such as truncated peptides. The identification of these novel peptides reflected the overall increased detection capability of our approach. Peptide fragments are commonly observed in various neuropeptidomics studies, even if strict sample preparation procedures are used. ${ }^{2,9,38-40}$ The formation of peptide fragments may come from in vivo peptide/protein processing, which are performed by intracellular proteolysis or by extracellular peptide decay after their release from neurons. ${ }^{41}$ These processes are not fully understood yet. However, a line of evidence showed that many peptide fragments have distinct physiological functions, for example, activating particular receptors or inhibiting enzymes. ${ }^{41,42}$ The identification of novel peptides therefore provided possible candidates for bioactive neuropeptides.

Our study allowed a number of hydrophobic and large peptides, including neuropeptide $\mathrm{Y}$ and galanin, identified from mouse brain. Neuropeptide $Y$ and galanin are of high interest in various biological studies due to their important roles such as food intake control and cognition. Although they are abundant in the brain, ${ }^{43}$ they have not been reported in previous neuropeptidomic studies with high confidence, probably because of its high hydrophobicity and long sequence. ${ }^{44}$ The reliable identification of such long and hydrophobic neuropeptides using our approach assists an exploration of their roles in physiological and behavioral processes. Moreover, our approach allowed these peptides quantified, if necessary, along with other hydrophilic peptides because the high coverage of peptides in a LC-MS analysis.

Taken together, we have described an integrated mass spectrometric approach for acquiring high identification rates of endogenous peptides from the mouse brain. Using the developed approaches, hydrophobic peptides as well as hydrophilic peptides could be simultaneously extracted from brain tissue. The subsequent CSD-LC-FT-MS/MS fully used the advantage of high accuracy FT-MS/MS while compensating for its slow scan speed. Moreover, the database search conducted at the sample level rather than individual raw data level simplified data processing and made the analysis much less time-consuming. The application of this approach to the mouse brain allowed us to identify an unrivaled number of endogenous peptides from neuropeptide precursor proteins. Our study presents a straightforward and powerful approach for the discovery of peptides from complex biological samples in discovery neuropeptidomics.

\section{- ASSOCIATED CONTENT}

S Supporting Information

Supplementary table and figures.

\section{AUTHOR INFORMATION}

\section{Corresponding Author}

*Phone: 0041-263008910. Fax: 0041-263009734. E-mail: xiaozhe.zhang@unifr.ch.

\section{Notes}

The authors declare no competing financial interest.

\section{ACKNOWLEDGMENTS}

We thank Prof. Robert Kretz and Dr. Barbara Becattini for brain sample preparation. We thank Dr. Vaclav Ranc and Dr. Jennifer Lynn Miles for comments and revision on the manuscript. This work was supported by the SNF R'Equip 316000-121308 and a EURYI award to G.R. P.E.A. acknowledges support from Swedish Research Council, Grants 2009- 
6050, 2008-5597, and 2010-5421, and the Knut and Alice Wallenberg Foundation.

\section{REFERENCES}

(1) Hokfelt, T.; Broberger, C.; Xu, Z. Q.; Sergeyev, V.; Ubink, R.; Diez, M. Neuropeptides-an overview. Neuropharmacology 2000, 39 (8), 1337-56.

(2) Svensson, M.; Skold, K.; Svenningsson, P.; Andren, P. E. Peptidomics-based discovery of novel neuropeptides. J. Proteome Res. 2003, 2 (2), 213-9.

(3) Rubakhin, S. S.; Romanova, E. V.; Nemes, P.; Sweedler, J. V. Profiling metabolites and peptides in single cells. Nat. Methods 2011, 8 (4 Suppl), S20-9.

(4) Fricker, L. D.; Lim, J.; Pan, H.; Che, F. Y. Peptidomics: identification and quantification of endogenous peptides in neuroendocrine tissues. Mass Spectrom. Rev. 2006, 25 (2), 327-44.

(5) Svensson, M.; Skold, K.; Nilsson, A.; Falth, M.; Nydahl, K.; Svenningsson, P.; Andren, P. E. Neuropeptidomics: MS applied to the discovery of novel peptides from the brain. Anal. Chem. 2007, 79 (1), $15-6$ and $18-21$.

(6) Altelaar, A. F.; Mohammed, S.; Brans, M. A.; Adan, R. A.; Heck, A. J. Improved identification of endogenous peptides from murine nervous tissue by multiplexed peptide extraction methods and multiplexed mass spectrometric analysis. J. Proteome Res. 2009, 8 (2), 870-6.

(7) Svensson, M.; Boren, M.; Skold, K.; Falth, M.; Sjogren, B.; Andersson, M.; Svenningsson, P.; Andren, P. E. Heat stabilization of the tissue proteome: a new technology for improved proteomics. $J$. Proteome Res. 2009, 8 (2), 974-81.

(8) Dowell, J. A.; Heyden, W. V.; Li, L. Rat neuropeptidomics by LC-MS/MS and MALDI-FTMS: Enhanced dissection and extraction techniques coupled with 2D RP-RP HPLC. J. Proteome Res. 2006, 5 (12), 3368-75.

(9) Lee, J. E.; Atkins, N. Jr.; Hatcher, N. G.; Zamdborg, L.; Gillette, M. U.; Sweedler, J. V.; Kelleher, N. L. Endogenous peptide discovery of the rat circadian clock: a focused study of the suprachiasmatic nucleus by ultrahigh performance tandem mass spectrometry. Mol. Cell. Proteomics 2010, 9 (2), 285-97.

(10) Petruzziello, F.; Fouillen, L.; Wadensten, H.; Kretz, R.; Andren, P. E.; Rainer, G.; Zhang, X. Extensive characterization of Tupaia belangeri neuropeptidome using an integrated mass spectrometric approach. J. Proteome Res. 2012, 11 (3), 886-96.

(11) Falth, M.; Skold, K.; Norrman, M.; Svensson, M.; Fenyo, D.; Andren, P. E. SwePep, a database designed for endogenous peptides and mass spectrometry. Mol. Cell. Proteomics 2006, 5 (6), 998-1005.

(12) Briand, L. A.; Gritton, H.; Howe, W. M.; Young, D. A.; Sarter, M. Modulators in concert for cognition: modulator interactions in the prefrontal cortex. Prog. Neurobiol. 2007, 83 (2), 69-91.

(13) Menschaert, G.; Vandekerckhove, T. T.; Baggerman, G.; Schoofs, L.; Luyten, W.; Van Criekinge, W. Peptidomics coming of age: a review of contributions from a bioinformatics angle. J. Proteome Res. 2010, 9 (5), 2051-61.

(14) Skold, K.; Svensson, M.; Norrman, M.; Sjogren, B.; Svenningsson, P.; Andren, P. E. The significance of biochemical and molecular sample integrity in brain proteomics and peptidomics: stathmin $2-20$ and peptides as sample quality indicators. Proteomics 2007, 7 (24), 4445-56.

(15) Zhu, X.; Desiderio, D. M. Methionine enkephalin-like immunoreactivity, substance P-like immunoreactivity and betaendorphin-like immunoreactivity post-mortem stability in rat pituitary. J. Chromatogr. 1993, 616 (2), 175-87.

(16) Skold, K.; Svensson, M.; Kaplan, A.; Bjorkesten, L.; Astrom, J.; Andren, P. E. A neuroproteomic approach to targeting neuropeptides in the brain. Proteomics 2002, 2 (4), 447-54.

(17) Che, F. Y.; Lim, J.; Pan, H.; Biswas, R.; Fricker, L. D. Quantitative neuropeptidomics of microwave-irradiated mouse brain and pituitary. Mol. Cell. Proteomics 2005, 4 (9), 1391-405.

(18) Rossbach, U.; Nilsson, A.; Falth, M.; Kultima, K.; Zhou, Q.; Hallberg, M.; Gordh, T.; Andren, P. E.; Nyberg, F. A quantitative peptidomic analysis of peptides related to the endogenous opioid and tachykinin systems in nucleus accumbens of rats following naloxoneprecipitated morphine withdrawal. J. Proteome Res. 2009, 8 (2), 10918.

(19) Kultima, K.; Nilsson, A.; Scholz, B.; Rossbach, U. L.; Falth, M.; Andren, P. E. Development and evaluation of normalization methods for label-free relative quantification of endogenous peptides. Mol. Cell. Proteomics 2009, 8 (10), 2285-95.

(20) Zhang, J.; Xin, L.; Shan, B.; Chen, W.; Xie, M.; Yuen, D.; Zhang, W.; Zhang, Z.; Lajoie, G. A.; Ma, B. PEAKS DB: De Novo sequencing assisted database search for sensitive and accurate peptide identification. Mol. Cell. Proteomics, 2011.

(21) Ma, B.; Zhang, K.; Hendrie, C.; Liang, C.; Li, M.; DohertyKirby, A.; Lajoie, G. PEAKS: powerful software for peptide de novo sequencing by tandem mass spectrometry. Rapid Commun. Mass Spectrom. 2003, 17 (20), 2337-42.

(22) Han, Y.; Ma, B.; Zhang, K. SPIDER: software for protein identification from sequence tags with de novo sequencing error. $J$. Bioinf. Comput. Biol. 2005, 3 (3), 697-716.

(23) Van Dijck, A.; Hayakawa, E.; Landuyt, B.; Baggerman, G.; Van Dam, D.; Luyten, W.; Schoofs, L.; De Deyn, P. P. Comparison of extraction methods for peptidomics analysis of mouse brain tissue. J. Neurosci. Methods 2011, 197 (2), 231-7.

(24) Cuttitta, F. Peptide amidation: signature of bioactivity. Anat. Rec. 1993, 236 (1), 87-93 and 172-3; discussion, 93-5.

(25) Eipper, B. A.; Stoffers, D. A.; Mains, R. E. The biosynthesis of neuropeptides: peptide alpha-amidation. Annu. Rev. Neurosci. 1992, 15, $57-85$.

(26) Nilsson, T.; Mann, M.; Aebersold, R.; Yates, J. R. 3rd; Bairoch, A.; Bergeron, J. J. Mass spectrometry in high-throughput proteomics: ready for the big time. Nat. Methods 2010, 7 (9), 681-5.

(27) Nilsson, A.; Falth, M.; Zhang, X.; Kultima, K.; Skold, K.; Svenningsson, P.; Andren, P. E. Striatal alterations of secretogranin-1, somatostatin, prodynorphin, and cholecystokinin peptides in an experimental mouse model of Parkinson disease. Mol. Cell. Proteomics 2009, 8 (5), 1094-104.

(28) Taylor, S. W.; Andon, N. L.; Bilakovics, J. M.; Lowe, C.; Hanley, M. R.; Pittner, R.; Ghosh, S. S. Efficient high-throughput discovery of large peptidic hormones and biomarkers. J. Proteome Res. 2006, 5 (7), 1776-84.

(29) Olsen, J. V.; de Godoy, L. M.; Li, G.; Macek, B.; Mortensen, P.; Pesch, R.; Makarov, A.; Lange, O.; Horning, S.; Mann, M. Parts per million mass accuracy on an Orbitrap mass spectrometer via lock mass injection into a C-trap. Mol. Cell. Proteomics 2005, 4 (12), 2010-21.

(30) An Thieu, V.; Kirsch, D.; Flad, T.; Muller, C.; Spengler, B. Direct protein identification from nonspecific peptide pools by highaccuracy MS data filtering. Angew. Chem., Int. Ed. 2006, 45 (20), 3317-9.

(31) Schmidt, A.; Gehlenborg, N.; Bodenmiller, B.; Mueller, L. N.; Campbell, D.; Mueller, M.; Aebersold, R.; Domon, B. An integrated, directed mass spectrometric approach for in-depth characterization of complex peptide mixtures. Mol. Cell. Proteomics 2008, 7 (11), 213850.

(32) Schmidt, A.; Claassen, M.; Aebersold, R. Directed mass spectrometry: towards hypothesis-driven proteomics. Curr. Opin. Chem. Biol. 2009, 13 (5-6), 510-7.

(33) Fountoulakis, M.; Hardmeier, R.; Hoger, H.; Lubec, G. Postmortem changes in the level of brain proteins. Exp. Neurol. 2001, 167 (1), 86-94.

(34) Franzen, B.; Yang, Y.; Sunnemark, D.; Wickman, M.; Ottervald, J.; Oppermann, M.; Sandberg, K. Dihydropyrimidinase related protein2 as a biomarker for temperature and time dependent post mortem changes in the mouse brain proteome. Proteomics 2003, 3 (10), 19209.

(35) Ferrer, I.; Santpere, G.; Arzberger, T.; Bell, J.; Blanco, R.; Boluda, S.; Budka, H.; Carmona, M.; Giaccone, G.; Krebs, B.; Limido, L.; Parchi, P.; Puig, B.; Strammiello, R.; Strobel, T.; Kretzschmar, H. Brain protein preservation largely depends on the postmortem storage temperature: implications for study of proteins in human neurologic 
diseases and management of brain banks: a BrainNet Europe Study. J. Neuropathol. Exp. Neurol. 2007, 66 (1), 35-46.

(36) Crecelius, A.; Gotz, A.; Arzberger, T.; Frohlich, T.; Arnold, G. J.; Ferrer, I.; Kretzschmar, H. A. Assessing quantitative post-mortem changes in the gray matter of the human frontal cortex proteome by 2 D DIGE. Proteomics 2008, 8 (6), 1276-91.

(37) Scholz, B.; Skold, K.; Kultima, K.; Fernandez, C.; Waldemarson, S.; Savitski, M. M.; Soderquist, M.; Boren, M.; Stella, R.; Andren, P.; Zubarev, R.; James, P. Impact of temperature dependent sampling procedures in proteomics and peptidomics-a characterization of the liver and pancreas post mortem degradome. Mol. Cell. Proteomics 2011, 10 (3), M900229MCP200.

(38) Sasaki, K.; Satomi, Y.; Takao, T.; Minamino, N. Snapshot peptidomics of the regulated secretory pathway. Mol. Cell. Proteomics 2009, 8 (7), 1638-47.

(39) Clynen, E.; Baggerman, G.; Veelaert, D.; Cerstiaens, A.; Van der Horst, D.; Harthoorn, L.; Derua, R.; Waelkens, E.; De Loof, A.; Schoofs, L. Peptidomics of the pars intercerebralis-corpus cardiacum complex of the migratory locust, Locusta migratoria. Eur. J. Biochem. 2001, 268 (7), 1929-39.

(40) Zhang, X.; Pan, H.; Peng, B.; Steiner, D. F.; Pintar, J. E.; Fricker, L. D. Neuropeptidomic analysis establishes a major role for prohormone convertase-2 in neuropeptide biosynthesis. J. Neurochem. 2010, 112 (5), 1168-79.

(41) Fricker, L. D. Analysis of mouse brain peptides using mass spectrometry-based peptidomics: implications for novel functions ranging from non-classical neuropeptides to microproteins. Mol. Biosyst. 2010, 6 (8), 1355-65.

(42) Xie, F.; London, S. E.; Southey, B. R.; Annangudi, S. P.; Amare, A.; Rodriguez-Zas, S. L.; Clayton, D. F.; Sweedler, J. V. The zebra finch neuropeptidome: prediction, detection and expression. BMC Biol. 2010, 8,28 .

(43) Balasubramaniam, A.; Sheriff, S.; Johnson, M. E.; Prabhakaran, M.; Huang, Y.; Fischer, J. E.; Chance, W. T. [D-TRP32]neuropeptide Y: a competitive antagonist of NPY in rat hypothalamus. J. Med. Chem. 1994, 37 (6), 811-5.

(44) Parkin, M. C.; Wei, H.; O’Callaghan, J. P.; Kennedy, R. T. Sample-dependent effects on the neuropeptidome detected in rat brain tissue preparations by capillary liquid chromatography with tandem mass spectrometry. Anal. Chem. 2005, 77 (19), 6331-8. 